\title{
Difficulties and possibilities in communication between referring clinicians and radiologists: perspective of clinicians
}

\author{
This article was published in the following Dove Press journal: \\ Journal of Multidisciplinary Healthcare
}

\author{
Nabi Fatahi ${ }^{1,2}$ \\ Ferid Krupic ${ }^{3}$ \\ Mikael Hellström ${ }^{1,4}$ \\ 'Department of Radiology, Institute of \\ Clinical Sciences, The Sahlgrenska \\ Academy, University of Gothenburg, \\ Gothenburg, Sweden; ${ }^{2}$ Department of \\ Learning and Leadership for Health Care \\ Professionals, Institute of Health and \\ Care Sciences, The Sahlgrenska Academy, \\ University of Gothenburg, Gothenburg, \\ Sweden; ${ }^{3}$ Department of Orthopaedics, \\ Institute of Clinical Sciences, The \\ Sahlgrenska Academy, University of \\ Gothenburg, Gothenburg, Sweden; \\ ${ }^{4}$ Department of Radiology, Sahlgrenska \\ University Hospital, Gothenburg, Sweden
}

Purpose: To investigate modes and quality of interprofessional communication between clinicians and radiologists, and to identify difficulties and possibilities in this context, as experienced by referring clinicians.

Patients and methods: Focus group interviews with 22 clinicians from different specialties were carried out. The leading question was: "How do you experience communication, verbal and nonverbal, between referring clinicians and radiologists?" Content analysis was used for interpretation of data.

Results: Overall, referring clinicians expressed satisfaction with their interprofessional communication with radiologists, and digital access to image data was highly appreciated. However, increased reliance on digital communication has led to reduced face-toface contacts between clinicians and radiologists. This seems to constitute a potential threat to bilateral feedback, joint educational opportunities, and interprofessional development. Cumbersome medical information software systems, time constraints, shortage of staff, reliance on teleradiology, and lack of uniform format of radiology reports were mentioned as problematic. Further implementation of structured reporting was considered beneficial.

Conclusion: Deepened face-to-face contacts between clinicians and radiologists were considered prerequisites for mutual understanding, deepened competence and mutual trust; a key factor in interprofessional communication. Clinicians and radiologists should come together in order to secure bilateral feedback and obtain deepened knowledge of the specific needs of subspecialized clinicians.

Keywords: interprofessional communication, referring physician, radiologist, face-to-face communication, radiology report, interprofessional trust

\section{Introduction}

Due to the rapid development of digital communication techniques during the last decades, ways of interprofessional communication in medicine have changed dramatically. This concerns all specialties, but is particularly evident in the communication between referring clinicians and radiologists. Thus, with the introduction of PACS (picture and archiving systems) and electronic referral and reporting systems, radiological images and reports are made available for clinicians anytime and anywhere, ie, not physically restricted to the radiology department or limited to consultations or demonstrations by radiologists at radiology rounds or conferences. Traditional and existing interprofessional communication may thereby be threatened,
Correspondence: Mikael Hellström Department of Radiology, Sahlgrenska University Hospital, 4I345 Gothenburg, Sweden

Tel +4673660I558

Email mikael.hellstrom@xray.gu.se 
eg, leading to fewer opportunities for clinicians and radiologists to communicate face-to-face with each other. ${ }^{1,2}$

The potential impact of this development on the quality and quantity of the bidirectional communication has been debated..$^{3-5}$ Poor communication among health care professionals has been identified as one of the primary causes of medical mistakes that adversely may affect patients' health. ${ }^{6-9}$ Thus, the quality of communication between referring clinicians and radiologists may have an impact on patient care and patient safety, and miscommunication may have medico-legal consequences. ${ }^{10}$ Gaining a better understanding of how referring clinicians communicate with radiologists is of importance not only for the care of the individual patient but also for the overall quality of health care. ${ }^{11,12}$

The aim of the present study was to investigate modes, quality and experiences of interprofessional communication between referring clinicians and radiologists, and to identify difficulties and possibilities in this context, as experienced by referring clinicians in a university hospital setting, working with digital imaging systems and electronic referral and reporting systems.

\section{Materials and methods Informants}

This is a qualitative study using data from interviews with clinical physicians (informants) from a university hospital. We performed four focus group discussions including a total of 22 physicians ( 9 women, 13 men), representing internal medicine, abdominal surgery and orthopedic surgery. The informants were recruited through announcements at their respective departments, on a voluntary basis. They were aged 32-63 years (median 41 years) and had 4-34 years' experience in their respective field.

\section{Ethical considerations}

The informants were given written and oral information of the aim and methods of the study. All informants gave their consent to participate. Participation was voluntary, and the informants could withdraw from the study at any time. All individual statements from the informants were anonymized and could not be traced to individual informants. The study contained no patient data. Formal ethical approval was not necessary for this type of study.

\section{Data collection}

Focus group discussions were performed in conference rooms outside the departments and were led by two of the three researchers (mediators).

The focus group discussions were semi-structured and began with the main questions "How do you experience communication, verbal and nonverbal, between the referring clinicians and the radiologists? What are the difficulties and possibilities? Are there barriers in communication, and can they be overcome?"

The initial questions were supplemented with short questions like "Could you tell us more about that?" The informants were encouraged to speak freely using their own words. Each group discussion lasted $>60$ mins. The discussions were digitally audio-recorded, with the informants' permission, and transcribed verbatim by a secretary, without knowledge of the identity of the informants.

\section{Data analysis}

Data analysis was performed by the three authors, all experienced in the methodology chosen. Qualitative content analysis method ${ }^{13}$ was used for analysis and interpretation of the data. The transcripts were read carefully by all researchers, for overview of the material. Thereafter, meaning units consisting of words, sentences or paragraphs containing aspects related to each other were extracted. Meaning units related to each other by content were grouped together into a condensed meaning unit, which was further abstracted and coded. Codes that addressed similar issues were grouped together, resulting in subcategories. Subcategories focusing on similar problems were brought together in categories and themes. The results are illustrated in the text by selected quotes.

\section{Results}

The analysis of the interviews resulted in three categories and seven subcategories (Table 1).

\section{Modes and quality of communication} The radiology report

The referring clinicians (informants) emphasized the role of the radiology report as vital for decision-making, quality assurance and patient safety. They also emphasized that its quality is essential for the trust between referring clinicians and radiologists.

The informants were generally satisfied with the quality of radiology reports. However, some expressed concern 


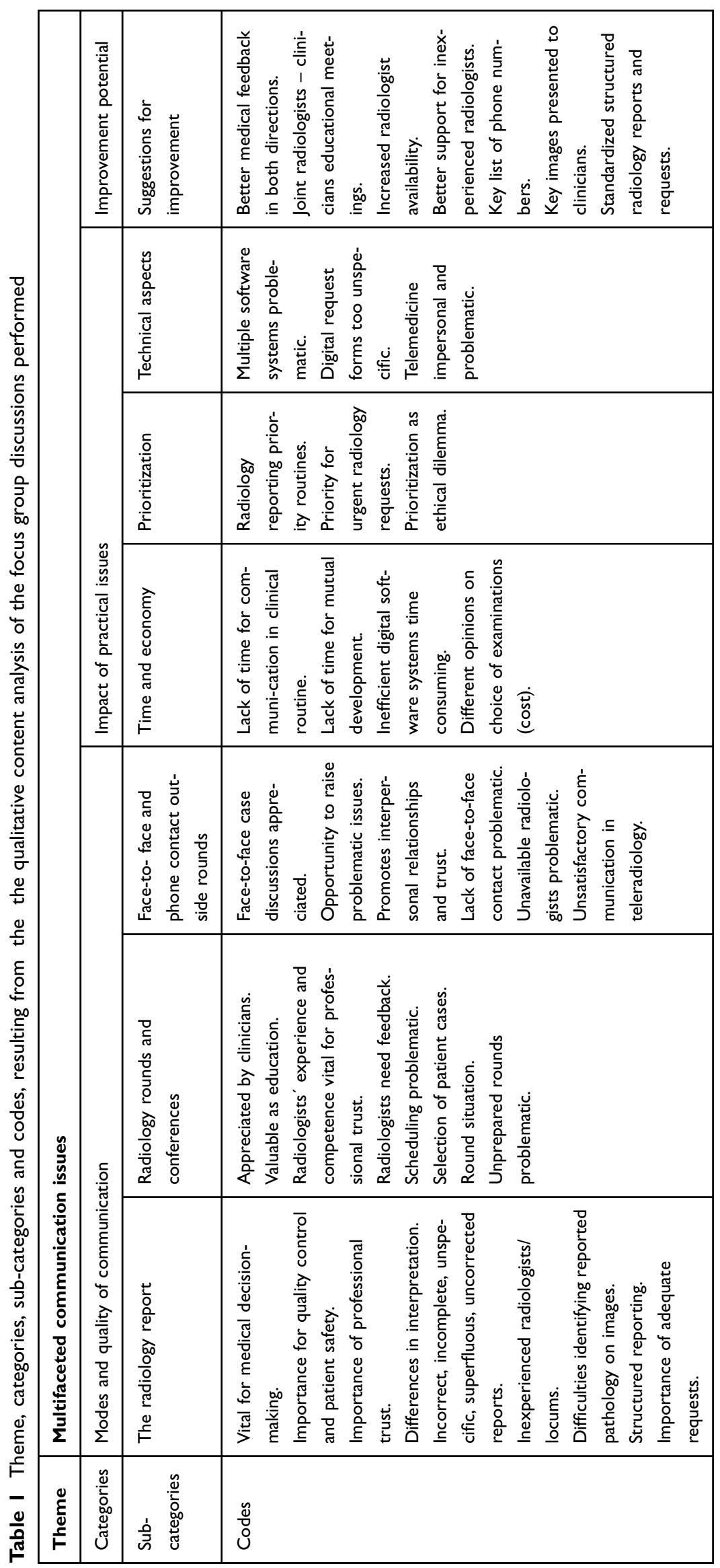


with the quality of reports from temporary locum radiologists at times of staff shortage, which were occasionally considered incorrect, incomplete or too unspecific. "If we ask two or three questions perhaps only one is answered and the rest remain unanswered". "The difficult thing with many new faces is that you don't know their competence".

The informants stated that reports using free text were sometimes too descriptive and too long: “... information drowns in a lot of text". Structured radiology reporting was not used routinely, but was advocated by the majority of informants, including templates agreed upon by clinicians and radiologists "... so that you know exactly which regions have been checked".

Another problem mentioned was radiology reports obtained via telemedicine: "We have many times received reports from external teleradiology units, but at our local conference they were not equivalent to the reports we received". When there was a discrepancy between the telemedicine report and the local report, the latter was considered more trustworthy. "It would be better if our own (radiologists) reported the cases, and we could come and discuss the images".

Another problem raised was the difficulty to effectively provide feedback in the digital communication system on reports that need adjustment after review by a clinician:

"It is troublesome when a radiology report is altered at second look and it is not documented". Clinicians were prone to make a note only in the medical file, which may be misleading if only radiology files are checked subsequently. It was also mentioned that incorrect reports are not always corrected in writing, because of concern for the colleague who made the report. "I don't want to step on his toes, he is so and so ..." ".

The informants also acknowledged that the quality and content of their own radiology requests could be improved: "I feel sometimes we do not write good enough request forms". It was suggested that a simple template sketch in the digital referral system of, eg, complex postoperative anatomy, would help the radiologists to understand the anatomy.

The informants also emphasized the need for deepened discussions between radiologists and referring clinicians on the content, form and structure of radiological reports, in order to maximize the usefulness of imaging data.

\section{Radiology rounds and conferences}

The informants stated that radiology rounds have been reduced in numbers after digital images and reports have been made easily accessible for clinicians outside radiology departments. The informants emphasized that radiology rounds and conferences are highly appreciated, and essential for both referring clinicians and radiologists, for patient care, problem solving, production control, feedback and education: "The thing with radiology rounds is that you meet so many, not only radiologists but also your colleagues (in your own field). It has an educational value for all involved". However, the individual experience and competence of the radiologist involved was highlighted as a deciding factor for the communication quality: "It depends on who holds the radiology round. Some are more experienced and the rounds will be a teaching moment, so that's great. Others may just take up what is already in the report".

The radiology round was considered a good opportunity for feedback to the radiologists. "Feedback to the radiologists is not so good, I think. On a radiology round, we can give feedback". On the other hand, clinicians sometimes seemed reluctant to convey negative feedback: "The risk is, if you don't know each other, that you appear nasty and just want to point out other people's mistakes". "In a busy clinical setting I may not prioritize giving feedback to a radiologist".

Practical issues, such as timing, patient selection and atmosphere of rounds and conferences were also considered important for the communication quality. Limited attendance of clinicians due to time constraints and insufficient time for radiologists to prepare rounds was mentioned as problematic.

\section{Face-to-face and phone contact outside rounds}

The informants stated that face-to-face communication and phone contact with the radiologists function very well. They specifically mentioned the benefit of viewing images together with the radiologists.

\footnotetext{
"Generally, I think we have a very good cooperation with radiologists. We have very good dialogue, and we know most of them who are permanent staff. In this way, we know whom we can approach, discuss and check the images with."
}

The informants mentioned the advantage of easy access to digital image information but pointed out that it has led to less frequent face-to-face contacts with the radiologists.

Unscheduled face-to-face radiologist consultations were much appreciated by the informants: 
"When I'm on call, I always visit the x-ray department and check cases together with the radiologist, because there are always cases to check and there is added value, not only medical, but also other value."

Another informant stated: "I experience face-to-face communication as very rewarding ... and there must be room for this in the radiologist's work schedule".

The need for face-to-face communication was emphasized by other informants: "It is more and more common that we want to discuss the images on the morning (of the surgery), for example in case of advanced cancer".

Lack of face-to-face contact was mentioned as problematic, eg, in case of teleradiology or other external reporting. "If we are serviced by radiologists abroad, it is virtually impossible to communicate". "We need a human being and the images in front of us to talk".

Telephone contact with the radiologists was generally well appreciated, especially in acute cases, and to sort out unclear referrals and reports: "If there is a controversial report I call the radiology department". "It goes the other way too, regarding the most acute cases that need immediate treatment". However, practical difficulties to reach the specific radiologist was mentioned as problematic,

"It is difficult because there is no direct number (on the report) to the radiologist in question. Then you have to call the x-ray department, and someone runs around looking for the radiologist, which takes time."

\section{Impact of practical issues \\ Time and economy}

The informants stated that communication between radiologists and referring clinicians in the clinical routine is limited by time constraints on both sides. "For us, it's very difficult to get this on the schedule. We're extremely hardpressed". It was stated that lack of time to physically visit radiology rounds and conferences and to consult radiologists individually makes learning and feedback between clinicians and radiologists difficult.

Lack of radiologists in certain segments of radiology, creating work over-load and time constraints was also mentioned as a factor that limits communication and learning: "I guess the experienced ones don't have time to train the newcomers".

Economy was mentioned as potential threat to interprofessional communication and trust, eg, when an ordered examination was exchanged to, or complemented with, another and more expensive examination, considered more appropriate by the radiologist. "Sometimes it can be a matter of costs. You don't want to add costs without prior discussion". Other informants were less concerned: "Conversion from x-ray of the neck to $\mathrm{CT}$ is frequently done without asking". "It's clear someone should pay, but for me it is not so interesting".

\section{Prioritization}

Prioritization is a necessity for referring clinicians as well as radiologists in settings with limited resources. "Acute cases tend to drown in the bulk of other acute referrals, because nearly everything from the emergency department is, by definition, acute". It was suggested that more discriminative levels of urgency on digital request forms would simplify prioritization for the radiologists.

The informants brought up the problem with patient waiting times and mentioned that in order to shorten waiting times, and thereby helping the patient, referring physicians might tend to exaggerate symptoms. "I think there is some overuse of the term acute (on the request form), not to risk a very long waiting time". This raised important ethical issues, but was not further discussed.

\section{Technical aspects}

Other issues concerned the digital medical information systems. These were praised for providing quick and safe delivery of requests, reports and images, facilitating communication between referring clinicians and radiologists. “... We have much better opportunities to view the images. It is an enormous advantage". However, limitations of the digital referral software system were also mentioned, eg, when trying to order examination of certain anatomical parts, eg, parts of a hand (finger) or foot (toe), when the system allowed only "hand" or "foot".

The major complaint regarding digital communication concerned the fact that at least three different computer systems, with different log-ins, different software structures and varying log-on waiting times were necessary to reach medical files, radiological images, laboratory data and other medical information in routine clinical work. "First you $\log$ in to one system and then to another. It takes many seconds each and you can just sit and watch". Other informants added: "We don't have fully integrated digital information systems. This takes extra time and causes frustration." "No one would buy such systems for private use". 


\section{Improvement potential - suggestions}

Apart from suggestions mentioned above, the informants suggested that there is potential for improved communication and understanding between referring clinicians and radiologists by extended knowledge sharing, joint educational activities and research collaboration. Educational activities should include deepened, joint discussions on what specific information the clinicians need and what information the radiologists can provide.

It was also suggested that feedback to the radiologists on their radiology reports should be facilitated in the digital communication systems.

The informants suggested that communication of radiological results may be facilitated by increased use of structured reporting, and by routinely implementing annotations and image numbers to mark relevant pathology in the image stack. "Because we can sit (alone) in front of 500 images and there are no arrows or other annotations to mark the pathology".

Another suggestion was for radiologists to be more clinically involved in the patients, to talk, observe and examine the patient in the x-ray department. "What we see is largely descriptive radiology, but clinical examinations by the radiologists is also needed". "I think it is important for the radiologist to come to the patient and ask where the pain is, and to palpate".

Referring to the difficulties with partly separate computer systems for clinical, radiological, laboratory and administrative patient information, the informants also emphasized the need for integrated computer systems with easy and quick access to medical data from different sources.

\section{Discussion}

Digital systems for communicating radiological information have led to less frequent personal contacts between clinicians and radiologists ${ }^{1,2}$ as indicated also in the present study. Our study raised a number of related issues concerning the modes and quality of communication between referring clinicians and radiologists, with implications for interprofessional trust, prioritization and, indirectly, patient care and safety. ${ }^{10}$

The ease by which clinicians can reach and view radiological images and reports is considered an enormous advantage, confirmed by the informants in the present study. Also knowing that once a radiology request form has been sent off in the digital system it has also reached the radiology department, was reassuring for the clinicians, considering that paper request forms may get lost or delayed on the way.

The informants emphasized the critical value of the written radiology report for clinical decision-making, quality control and patient safety. Generally, they were satisfied with the quality of reports, but a slightly more critical view on radiologists 'competence could be traced from the orthopedic surgeons, as compared to abdominal surgeons and internists. This could possibly be explained by the fact that orthopedic surgeons are more likely to analyze images themselves in their daily routine, and thereby may be more competent, and perhaps more critical, than abdominal surgeons and internists, who rely more heavily on the radiologists. Specifically, in periods of understaffing, an increased number of reports from temporary locum radiologists with limited experience were considered unspecific, incomplete or incorrect. This was considered a potential threat to the interprofessional trust, and clinicians had to rely more on their own image interpretations. It also meant loss of quality control, as even experienced clinicians need support from radiologists, as claimed by the informants. It should be emphasized, however, that such understaffing at the time of the study was exceptional and temporary. Nevertheless, it shows the vulnerability of the system, in an era of shortage of qualified radiologists.

Opportunities for feedback face-to-face have diminished with increased digitalization, reduction of radiological rounds and increased use of telemedicine. Our informants often considered providing feedback to radiologists too time-consuming. For example, suboptimal reports could remain unchanged in the digital reporting system, while a separate note may have been made in the medical file (in a different computer system) by the clinician. Even at radiological rounds and conferences, some clinicians seemed hesitant to give "negative" feedback, not to appear "nasty", especially when "you don't know each other". Feedback from clinicians, surgery and pathology is vital for radiologists to maintain and develop diagnostic skills, while lack of feedback may threaten patient safety. Conveying feedback to radiologists should therefore be facilitated as part of normal routines.

Radiology needs to add significant value to the clinician and the patient. ${ }^{14}$ The need for optimized quality and utility of radiology reports was emphasized in our study, as previously highlighted. ${ }^{15}$ Some informants described the radiology reports as merely descriptive, and some suggested a more clinical approach, where the radiologist talks to, observes and examines the patient. 
The informants stated that they are sometimes left with a written report together with hundreds or thousands of digital CT or MR images, but without image annotations to show the pathology. Applying such annotations routinely, linked to the written report, would greatly facilitate communication of the message from the radiologist.

Another potential improvement suggested by the informants was increased use of structured reporting, ie, defined structure, format and style of the report, using defined terms and language to describe findings. Arguments for structured reporting are that free-text reports are not always read or understood, do not always answer all questions and do not allow easy data retrieval for quality control, research and teaching. Using free-text, clinician's' response to a report may be very different. ${ }^{16}$ Structured reporting has been shown to improve diagnostic accuracy ${ }^{17-19}$ and is used increasingly, but has yet to be widely implemented.

The quality of the radiology report is not only dependent on the ability of the radiologist, but also on the information given by the referring clinician on the request form. In a sense of self-criticism, this was brought up by some informants, suggesting that there is room for improvement on both the sender and receiver sides of the radiology request form.

The informants expressed that communication at rounds and conferences is appreciated, and essential for bilateral feed-back on cases, patient care and quality control, as emphasized by others. ${ }^{14,20,21}$ They also pointed at the experience and expertise of the participants involved, and the importance of allowing the radiologists enough scheduled time for round preparation, to optimize the outcome. In a previous study, radiologists identified late arrival of clinicians, lack of attention and disturbing phone calls during the rounds as problematic. ${ }^{20}$ Adequate scheduling and mutual agreements on conduct codes at rounds and conferences should be ascertained for the benefit of both parties.

Other valuable aspects of rounds and conferences mentioned were gaining knowledge on strengths and limitations of radiological methods, while radiologists at the same time gain important clinical knowledge, which may improve diagnostic performance.

Opinions revealed in our study are supported by studies showing that in-person communication between radiologists and clinicians adds significant clinical value, even when the original written report contains all necessary data. $^{22}$
Despite the development of advanced digital communication systems, personal contacts between referring clinicians and radiologists were considered essential for the interprofessional communication. Not only rounds and conferences, but also individual radiology consultations were highly appreciated. In the light of decreasing number of scheduled contacts between clinicians and radiologists, it was considered important that radiologists are allowed time for such consultations. Informal consultations for quick discussions on, eg, examination results were mentioned as highly effective, although difficulties to reach the responsible radiologist were mentioned as problematic. Such informal consultations may modify the interpretation of the radiological diagnostic information in a considerable number of cases, which may, or may not, be noted by the referrer in the medical file, while a corresponding note in the radiology system is rarely done,$^{23}$ a potential medico-legal and patient safety issue.

In an interesting medical anthropological study approach, Tillack and Breiman found that referring physicians mainly consult radiologists with whom they already have a personal relationship. ${ }^{2}$ This underscores the importance of building personal relationships for trust and understanding between referring physicians and radiologists. Interestingly, similar views were expressed by radiologists when asked about their communication with referring physicians. ${ }^{20}$ Active steps should thus be taken to deepen such contacts. One informant experienced the increased value of visiting the radiology department during on-calls not only to discuss cases, but also for a moment of informal socializing. Such informal contacts between radiologists and referring physicians may be an underestimated source of trust-building that should be further explored. Tillack and Borgstede found that radiology reading rooms embedded in different clinical departments, rather than located in the radiology department, led to more visits from the clinicians, and thereby more referring clinician - radiologist interactions. ${ }^{24}$ However, their findings also suggested that the communication patterns may differ between different groups of physicians and radiologists. Although radiology reading rooms embedded in a clinical environment may be beneficial for communication between radiologists and referring physicians, it may lead to isolation of radiologists from their own colleagues. Thus, radiologists also need to communicate with each other, in order to consult with more experienced colleagues, solve difficult cases and to give support to residents. 
When clinicians are distant from the radiology department, or radiologists are reporting from a remote place, physical face-to-face communication may be impossible. Telemedicine communication is then a possibility, but as highlighted in the present study, communication often fails in clinical practice due to difficulties to reach the radiologist and lack of interactivity while discussing the images. Teleradiology has gained increased interest as a complement or substitute for regular radiology service at many hospitals. ${ }^{25}$ It is considered to reduce costs for radiology staff locally, eg, on-call night service, which can be offered from teleradiology centers working day-time in different time zones. Immediate availability of reports irrespective of the time of the day or night is appealing, but many clinicians in the present study considered teleradiology as problematic, as it was felt to be impersonal, not knowing the reporting radiologist. Also, less trust was put into teleradiology reports, as compared to reports produced locally, especially when local reports are delivered together with image review at individual consultations, rounds or conferences. It seems to be of great value for clinicians to know the reporting radiologist, and to be able to discuss the images face-to-face, thereby developing interprofessional trust. ${ }^{2,22,26}$ Reduced interactions between referring clinicians and the radiologist working at a distance may lead to isolation, and even a feeling of loneliness of the radiologist, as pointed out by Gunderman and Tillack. ${ }^{27}$ This type of communication may potentially be improved by so-called virtual consultation, when the clinician and the distant radiologist communicate by a realtime computer screen share system. ${ }^{27}$ This allows both parts to simultaneously and interactively view the images and control the display, both being able to point to interesting pathology, while chatting digitally or talking on the phone. Gunderman and Tillack suggested that "If PACS has isolated us (radiologists) from referring physicians, we can use PACS to reconnect". ${ }^{27}$ Apart from more efficient ways of real-time audio-and-video communications, ${ }^{28}$ adding photographs of referring physicians and reporting radiologists on requests and reports could make communication more personalized, in order to build trust. ${ }^{27}$

Face-to-face and telephone contacts between referring clinicians and radiologists were thus considered important to promote interprofessional trust. The question of trust could be traced as a latent, but important, theme in the present study, hidden behind many of the opinions, critics and suggestions provided by the informants. The suggestions to increase joint organizational, educational and research activities can also be seen as efforts to build trust. ${ }^{29,30}$ Foreseeing an even stronger digital impact on imaging sciences by, eg, artificial intelligence, it seems important not to underestimate the role of human values, including interprofessional communication and trust, in this development.

Our study indicated that lack of time is a factor that significantly affects the quality of interprofessional communication, as shown previously. ${ }^{31,32}$ In addition to time factors mentioned above, lack of time for joint meetings to discuss indications, examination protocols and administrative issues were all mentioned as factors that negatively influenced interprofessional communication.

With limited health care resources, there are often waiting times for radiological examinations. Therefore, both referring clinicians and radiologists need to prioritize among patients. The information on the radiology request is usually the only patient information available for the radiologist, who has to decide which patient should be examined first. The informants were concerned about the tendency among referring physicians to exaggerate the patient's symptoms in order to shorten waiting time. This has ethical implications and has previously been highlighted by radiologists ${ }^{20}$ and should be the subject of further interprofessional discussions, in order to avoid unequal treatment of patients who may not get the right priority.

\section{Study limitations}

Our study was limited to opinions of clinicians from specialties in a university hospital setting, and their experiences may not be fully representative for other hospitals or other clinical specialties. In order to get broad views on referring physicians' communication with radiologists, we chose specialties with rich contacts with radiology, but with different levels of autonomy in interpreting radiological images, and thereby different levels of reliance on radiologists. The different groups of physicians (informants) were interviewed in separate focus groups. We chose this approach to stimulate a free, open climate and make the informants feel comfortable. As one of the researchers acting as a mediator was a radiologist, openness during the focus group discussion may have been affected when discussing the communication with radiologists. Thus, the informants' awareness of the background of the mediators might have influenced their willingness to bring up certain issues, out of fear of being impolite, blunt or offensive toward the mediator's department. On the 
other hand, it might also have been considered by the informants to be a unique opportunity to convey criticism or valuable tips that may improve communication between clinicians and radiologists. As mediators, we experienced that the atmosphere during the focus group discussions was open, honest and uninhibited. In the interpretation of the focus group data, the background of the researchers and their level of pre-understanding may also be a source of bias. ${ }^{33}$ This risk was minimized by including two nonradiologists in the data interpretation, and by the very awareness of such potential bias.

Opinions expressed from the informants could also have been biased by a temporary lack of radiology staff in one radiology section during the study period, although this situation probably is not unique. Finally, the number of interviewed physicians was limited. However, the transcribed interviews were rich in content and considered suitable for the content analysis method used, allowing manifest as well as latent content to be analyzed. ${ }^{13}$

\section{Conclusion}

Knowledge about how referring physicians experience communication with radiologists and radiology departments is fundamental for the future development of imaging services, and consequently for diagnostic quality and patient safety. Overall, referring clinicians expressed satisfaction with their communication with radiologists, and the easy digital communication of image data. However, increased reliance on digital communication has led to reduced face-to-face contacts between clinicians and radiologists. This constitutes a potential threat to bilateral feedback, joint educational opportunities and interprofessional development. Cumbersome medical information software systems, time constraints, shortage of radiology staff, reliance on teleradiology and lack of uniform format of radiology reports were mentioned as problem areas. Further implementation of structured reporting was considered beneficial. Most important, increased face-to-face contacts between clinicians and radiologists were considered a prerequisite for mutual understanding, deepened competence and mutual trust - a key factor in interprofessional communication. Ways to maintain and improve face-to-face contacts between referring physicians and radiologists should be sought.

\section{Acknowledgments}

The study was financially supported by the Department of Radiology at the Sahlgrenska University Hospital in
Gothenburg, and by grants from the Swedish State under the agreement between the Swedish Government and the country councils, the so called ALF-agreement (Avtal om läkarutbildning och forskning).

\section{Disclosure}

The authors report no conflicts of interest in this work.

\section{References}

1. Reiner B, Siegel E, Protopapas Z, Hooper F, Ghebrekidan H, Scanlon M. Impact of filmless radiology on frequency of clinician consultations with radiologists. AJR Am J Roentgenol. 1999;173:1169-1172. doi:10.2214/ajr.173.5.10541082

2. Tillack AA, Breiman RS. Renegotiating expertise: an examination of PACS and the challenges to radiology using a medical anthropologic approach. J Am Coll Radiol. 2012;9:64-68. doi:10.1016/j. jacr.2011.07.006

3. Reiner B. Automating radiologist workflow, part 1: the digital consultation. J Am Coll Radiol. 2008;5:1080-1085. doi:10.1016/j. jacr.2008.05.014

4. Baker S. Transmission of nonemergent critical findings: communication versus consultation. Radiology. 2010;257:609-611. doi:10.1148/ radiol.10101679

5. Margulis A. The constantly changing field of radiology: maintaining professionalism in an era of electronic communication. Radiology. 2010;257:22-23. doi:10.1148/radiol.10100691

6. Woolf S, Kuzel A, Dovey S, et al. A string of mistakes: the importance of cascade analysis in describing, counting, and preventing medical errors. Ann Fam Med. 2004;2:317-326.

7. Lingard L, Espin S, Whyte G, et al. Communication failures in the operating room: an observational classification of recurrent types and effects. Qual Saf Health Care. 2004;13:330-334. doi:10.1136/ qhe. 13.5 .330

8. Leonard M, Graham S, Bonacum D. The human factor: the critical importance of effective teamwork and communication in providing safe care. Qual Saf Health Care. 2004;13:85-90. doi:10.1136/ qshc.2004.010033

9. Reiner BI. Innovation opportunities in critical results communication: theoretical concepts. J Digit Imaging. 2013;26:605-609. doi:10.1007/ s10278-013-9609-4

10. Pinto F, Capodieci G, Setola FR, et al. Communication of findings of radiologic examinations: medicolegal considerations. Semin Ultrasound CT MR. 2012;33:376-378. doi:10.1053/j. sult.2012.01.014

11. Eisenberg R, Yamada K, Yam C, et al. Electronic messaging system for communicating important, but nonemergent, abnormal imaging results. Radiology. 2010;257:724-731. doi:10.1148/radiol.10101015

12. Rider EA, Kurtz S, Slade D, et al. The international charter for human values in healthcare: an interprofessional global collaboration to enhance values and communication in healthcare. Patient Educ Couns. 2014;96:273-280. doi:10.1016/j.pec.2014.06.017

13. Graneheim UH, Lundman B. Qualitative content analysis in nursing research: concepts, procedures and measures to achieve trustworthiness. Nurse Educ Today. 2004;24:105-112. doi:10.1016/j. nedt.2003.10.001

14. Lesslie MD, Parikh JR. Multidisciplinary tumor boards: an opportunity for radiologists to demonstrate value. Acad Radiol. 2017;24:107110. doi:10.1016/j.acra.2016.09.006

15. Plumb AA, Grieve FM, Khan SH. Survey of hospital clinicians' preferences regarding the format of radiology reports. Clin Radiol. 2009;64:386-394. doi:10.1016/j.crad.2008.11.009 
16. Rosenkrantz AB. Differences in perceptions among radiologists, referring physicians, and patients regarding language for incidental findings reporting. Am J Roentgenol. 2017;208:140-143. doi:10.2214/AJR.16.16633

17. Schoeppe F, Sommer WH, Nörenberg D, et al. Structured reporting adds clinical value in primary CT staging of diffuse large B-cell lymphoma. Eur Radiol. 2018;28:3702-3709. doi:10.1007/s00330-018-5340-3

18. Franconeri A, Fang J, Carney B, et al. Structured vs narrative reporting of pelvic MRI for fibroids: clarity and impact on treatment planning. Eur Radiol. 2018;28:3009-3017. doi:10.1007/s00330-017-5161-9

19. Magnetta MJ, Donovan AL, Jacobs BL, Davies BJ, Furlan A. Evidence-based reporting: a method to optimize prostate MRI communications with referring physicians. Am $J$ Roentgenol. 2018;210:108-112. doi:10.2214/AJR.17.18260

20. Fatahi N, Krupic F, Hellström M. Quality of radiologists' communication with other clinicians - as experienced by radiologists. Patient Educ Couns. 2015;98:722-727. doi:10.1016/j.pec.2015.02.009

21. Fefferman NR, Strubel NA, Prithiani C, Chakravarti S, Caprio M, Recht MP. Virtual radiology rounds: adding value in the digital era. Pediatr Radiol. 2016;46:1645-1650. doi:10.1007/s00247-016-3675-y

22. Dickerson EC, Alam HB, Brown RK, Stojanovska J, Davenport MS. In-person communication between radiologists and acute care surgeons leads to significant alterations in surgical decision making. $J$ Am Coll Radiol. 2016;13:943-949. doi:10.1016/j.jacr.2016.02.005

23. Won E, Rosenkrantz AB. Informal consultations between radiologists and referring physicians, as identified through an electronic medical record search. Am J Roentgenol. 2017;209:965-969. doi:10.2214/AJR.17.18050

24. Tillack AA, Borgstede JP. An evaluation of the impact of clinically embedded reading rooms on radiologist-referring clinician communication. J Am Coll Radiol. 2013;10:368-372. doi:10.1016/j.jacr.2012.12.009
25. Hryhorczuk AL, Hanneman K, Eisenberg RL, Meyer EC, Brown SD. Radiologic professionalism in modern healthcare. Radiographics. 2015;35:1779-1788. doi:10.1148/rg.2015150041

26. Coppola F, Bibbolino C, Grassi R, et al. Results of an Italian survey on teleradiology. Radiol Med. 2016;121:652-659. doi:10.1007/ s11547-016-0640-7

27. Gunderman RB, Tillack AA. The loneliness of the long-distance radiologist. J Am Coll Radiol. 2012;9:530-533. doi:10.1016/j. jacr.2011.12.021

28. Rosenkrantz AB, Sherwin J, Prithiano CP, Ostrow D, Recht MP. Technology-assisted virtual consultation for medical imaging. $J$ Am Coll Radiol. 2016;13:995-1002. doi:10.1016/j.jacr.2016. 02.029

29. Friberg K, Husebø SE, Olsen ØE, Saetre Hansen B. Interprofessional trust in emergency department - as experienced by nurses in charge and doctors on call. J Clin Nurs. 2016;25:3252-3260. doi:10.1111/ jocn. 13359

30. Gregory PA, Austin Z. Trust in interprofessional collaboration: perspectives of pharmacists and physicians. Can Pharm J. 2016;149:236-245. doi:10.1177/1715163516647749

31. Fatahi N, Hellström M, Skott C, Mattsson B. General practitioners' views on consultations with interpreters: a triad situation with complex issues. Scand J Prim Health Care. 2008;26:40-45. doi:10.1080/ 02813430701877633

32. Fatahi N, Mattsson B, Hasanpoor J, Skott C. Interpreters' experiences of general practitioner-patient encounters. Scand J Prim Health Care. 2005;23:159-163. doi:10.1080/02813430510018509

33. Nyström M, Dahlberg K. Pre-understanding and openness - a relationship without hope? Scand J Caring Sci. 2001;15:339-346.

\section{Publish your work in this journal}

The Journal of Multidisciplinary Healthcare is an international, peerreviewed open-access journal that aims to represent and publish research in healthcare areas delivered by practitioners of different disciplines. This includes studies and reviews conducted by multidisciplinary teams as well as research which evaluates the results or conduct of such teams or healthcare processes in general. The journal covers a very wide range of areas and welcomes submissions from practitioners at all levels, from all over the world. The manuscript management system is completely online and includes a very quick and fair peer-review system. Visit http://www.dovepress.com/testimonials. php to read real quotes from published authors. 